\title{
Exposure to Sleep, Rest, or Exercise Impacts Skill Memory Consolidation but so Too Can a Challenging Practice Schedule
}

\author{
두aewon Kim, ${ }^{1}$ and David L. Wright ${ }^{2}$
}

https://doi.org/10.1523/ENEURO.0198-21.2021

${ }^{1}$ Department of Neurology, Duke University School of Medicine, Durham, NC 27710 and ${ }^{2}$ Department of Kinesiology, Texas A\&M University, College Station, TX 77845

\begin{abstract}
When discussing procedural learning, it is now routine to consider both online and offline influences for skill acquisition. This is because it is commonly assumed that the evolution of a novel skill memory continues well after practice is over. Indeed, factors impacting offline contributions to skill memory development such as sleep and exercise have garnered considerable research interest in recent years. This is partly because of their capacity to foster postpractice consolidation, a process that has been identified as critical to moving a skill memory from a labile to more stable or elaborate form. While uncovering the potency of non-practice factors to facilitate consolidation is undoubtedly important, the present opinion is designed to remind the reader that a practice schedule, organized to challenge the learner, can, in and of itself, be effective in supporting consolidation resulting in significant gains in long-term skill retention.
\end{abstract}

Key words: consolidation; contextual interference; interleaved practice; motor learning; procedural memory

\section{Significance Statement}

Adopting "best practices" is an objective for training and rehabilitation design and typically focuses on practice composition. Recent work has championed postpractice activities, such as sleep and exercise, as critical to the evolution of skill memory. Such practice adjuncts presumably facilitate skill acquisition by improving memory consolidation. The present opinion highlights the potency of practice organization as a means to also improve postpractice consolidation illustrated by findings revealing that experience with an interleaved rather than repetitive practice (IP) format results in performance gains for up to $72 \mathrm{~h}$ following the conclusion of training. Such latent gains are associated with heightened primary motor cortex (M1) excitability following practice in a manner similar to that observed when supplementing practice with sleep or exercise.

Received May 3, 2021; accepted July 19, 2021; First published August 31, 2021.

The authors declare no competing financial interests. Author contributions: T.K. and D.L.W. wrote the paper.

Acknowledgements: We thank an anonymous reviewer.

Correspondence should be addressed to David L. Wright at davidwright@ tamu.edu.

https://doi.org/10.1523/ENEURO.0198-21.2021

Copyright (c) $2021 \mathrm{Kim}$ and Wright

This is an open-access article distributed under the terms of the Creative Commons Attribution 4.0 International license, which permits unrestricted use, distribution and reproduction in any medium provided that the original work is properly attributed.

\section{Fostering Skill Memory through Rest, Sleep, and Exercise}

Most accept that the evolution of a skill memory continues long after practice is over (Robertson and Takacs, 2017). Early efforts to look beyond the boundaries of practice to finds ways to enhance retention of a new skill focused almost exclusively on unique contributions from sleep and wake periods (Robertson et al., 2004). Indeed, a recent report of micro-consolidation during skill acquisition has reignited interest in the rest period during wake as the temporal locus of critical memory processes 
central to learning even when the interval is just a few seconds (Bonstrup et al., 2019). More recently, a quite different intervention, a brief bout of cardiovascular exercise, has also been touted as a powerful supplement to practice capable of procuring latent gains in skill (Jo et al., 2019; Chen et al., 2020).

In general, facilitation in skill memory following a sufficient rest period, exposure to sleep, and/or exercise is proposed to result from improved memory consolidation. The behavioral manifestation of successful consolidation is preservation of performance, even in the face of interference, or a gain in performance across a significant time period despite no additional practice. A vast body of evidence exists that reveals a distinction between consolidation that is sleep or time dependent leading to significant gains for a wide-range of procedural skill memories. In the case of sleep, novel skill memories can be facilitated when privy to either overnight sleep or a daytime nap (for recent review, see King et al., 2017). In contrast, the case for using acute exercise as a mediator of postpractice consolidation is still in relative infancy. Despite this, evidence is currently available supporting the claim that a brief bout of vigorous cardiovascular activity can protect a newly acquired procedural memory from interference from subsequent procedural (Jo et al., 2019) or declarative (Chen et al., 2020) learning that occurs in close temporal proximity. Moreover, when the interfering activity is absent, the administration of an acute bout of exercise immediately after novel skill learning, rather than an equivalent period of rest, has been reported to enhance skill memory assessed across an 8-h wake period (Ostadan et al., 2016).

\section{Increasing Interference during Practice Can Facilitate Consolidation of Novel Skill Memories}

While the use of sleep and exercise as adjuncts to practice continue to capture the research community's attention, the importance of practice itself, arguably the most critical determinant of the development of skill memory, has taken a relative backseat. The present commentary is an attempt to remind the reader that organizing practice of a novel skill can also be a conduit to continued processing of a newly formed skill memory well beyond the conclusion of a bout of physical practice. In the present case, the potency of one particular practice organization for encouraging postpractice consolidation is highlighted by briefly reviewing some recent data addressing contextual interference $(\mathrm{Cl})$, a practice scheduling phenomenon that has a rich history in the motor skill literature (Wright et al., 2016). Thus, rather than manipulate the postpractice period directly (i.e., by experiencing sleep or exercise after practice), here we demonstrate that the arrangement of practice can be such that postpractice memory processing is encouraged that can lead to sizeable latent gains.

Experimental evidence from studies conducted under the rubric of $\mathrm{Cl}$ underscore the importance of how practice organization can influence the resultant long-term retention of newly acquired skill memories. The $\mathrm{Cl}$ phenomenon has most commonly focused on learning outcomes that result from the simultaneous acquisition of multiple novel procedural skills in either an interleaved practice (IP) or repetitive practice (RP) format (Fig. 1A,B; Wright et al., 2016). IP is argued to induce greater demand on motor planning operations during training because of the frequent change in the trial-to-trial task demands that are encountered by the learner during this practice format (Li and Wright, 2000). Specifically, when an individual learns in the context of IP, they rarely, if ever, execute the same procedural skill on two consecutive practice trials within a single bout of training. Conversely, RP, a commonly adopted protocol in many instructional and rehabilitation settings, is argued to create relatively less interference because it involves the repeated execution of the same motor task before encountering any practice with other motor tasks that will also be practiced, and hopefully learned, during a single practice episode.

It turns out that IP, while characterized by poorer encoding reflected in a relatively slower rate of skill acquisition but greater attention demand assessed using dualtask procedures (Li and Wright, 2000), is more effective for supporting long-term retention of novel skill memory most often assessed $24 \mathrm{~h}$ after practice is over (Shea and Morgan, 1979; for limitations of IP, also see Wulf and Shea, 2002; Russell and Newell, 2007). Of particular interest for the present discussion is the observation that individuals exposed to IP, much like the participants privy to exercise or sleep following practice, exhibit a dramatic improvement in performance across a 24- to 72-h test interval despite the absence of any extra physical practice, that is, they display offline enhancement (Lin et al., 2011; Wright et al., 2016; Fig. 1C). What makes this gain even more impressive is that it emerges even when the test context for IP-trained learners is incongruent with the practice format that was experienced during original learning. That is, the gains after practice are equally likely to surface if the test of skill memory is administered in a RP or IP format. For example, recent work revealed more than a $20 \%$ loss in performance $48 \mathrm{~h}$ after the conclusion of RP compared with a $>30 \%$ gain in performance over the same interval following IP when test trials were administered in a RP schedule (Kim et al., 2018). Thus, skill memories resulting from exposure to IP are not only enhanced but are quite robust to a change in performance context. On the other hand, participants that experience $\mathrm{RP}$, despite exposure to the same total number of practice trials within a single training episode exhibit significantly better performance when the test environment matches that encountered during acquisition (Lin et al., 2011).

The robustness of skill learning following IP as opposed to $\mathrm{RP}$ is further highlighted by findings that demonstrate that the offline gains fostered by IP exhibit some resistant to interference from supplemental practice with another novel procedural skill. Specifically, despite the additional practice with a new procedural skill that began 5 min after IP was completed, participants still managed to exhibit performance $24 \mathrm{~h}$ later that was similar to that achieved at the end of training. This was not the case for their RP 
A

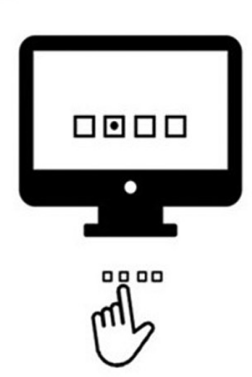

E

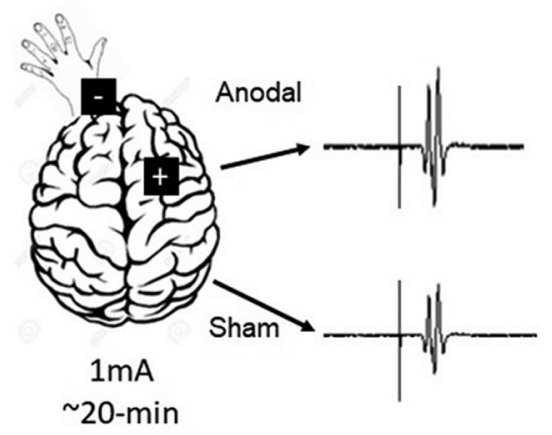

B

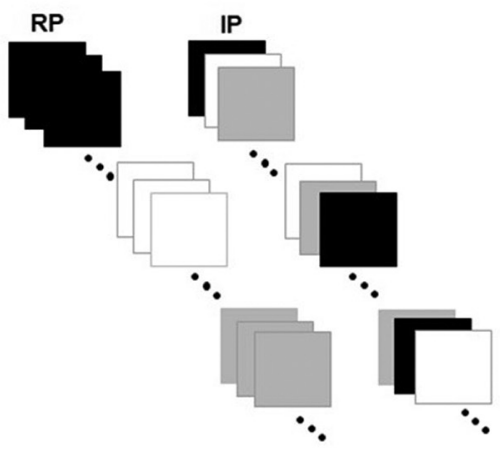

C

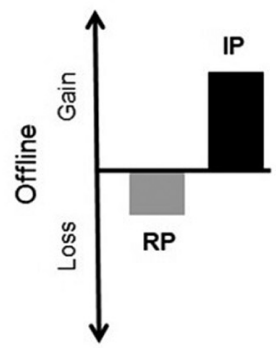

D

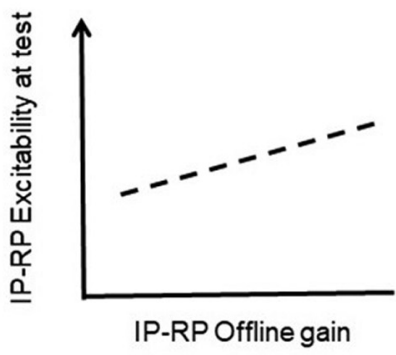

F

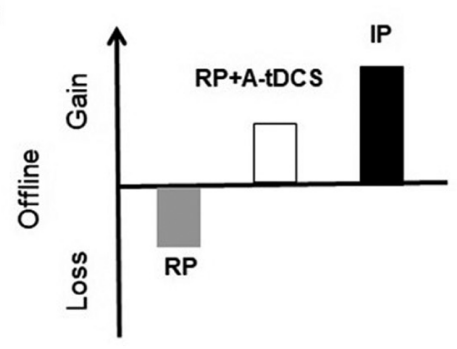

Figure 1. A motor sequence task is a common procedural skill used to reveal offline performance gains. This task requires the performer execute a series of key presses that are spatially compatible with visual signals presented on a display as quickly and accurately as possible $(\boldsymbol{A})$. A number of motor sequences (trials for three unique sequences, represented in black, gray, or white, are used for the purpose of illustration in this example) are practiced in either a repetitive (RP) or interleaved (IP) format during a period of training. For RP, all trials of practice for one of the sequences are completed during a period of training before any practice with an alternative motor sequence is introduced. Alternatively, in IP, during any period of practice, an equal number of trials for all of the to-be-learned sequences are experienced. However, the presentation of trials of practice for these to-be-learned sequences rarely, if ever, involve the execution of the same sequence on two consecutive trials within a single bout of training. The impact on performance following each of these training contexts is most commonly assessed during a delayed test usually administered $24-72 \mathrm{~h}$ later (not shown in diagram; Lin et al., 2011; Wright et al., 2016; $\boldsymbol{B}$ ). Offline gain, defined as the difference between performance at the time of test and at the end of training in either a IP or RP format, is greater following IP compared with RP (Wright et al., 2016; Lin et al., 2011; C), and the difference in offline gain achieved following RP and IP is related to the difference in cortical excitability observed at M1 before a test administered $72 \mathrm{~h}$ after practice was completed between individuals assigned to IP and RP (Lin et al., 2011 ; D). tDCS involves the passage of direct current between two electrodes [anode (+), cathode (-)]. Anodal stimulation (e.g., $1 \mathrm{~mA}$ for $20 \mathrm{~min}$ ) is a condition in which the anode is placed over the neural region being targeted resulting in an upregulation of activity at the stimulated site (e.g., M1) reflected in an increased MEP from the application of single-pulse TMS compared with sham stimulation $(\boldsymbol{E})$. Applying anodal tDCS at right M1 while the learner performed a motor sequence task with the left hand during RP results in offline gains rather than the anticipated loss in performance commonly observed following RP when performed in the absence of stimulation (Kim and Wright, 2020; $\boldsymbol{F}$ ).

counterparts who revealed substantial degradation in performance across the same timeframe (Kim et al., 2016). These data are reminiscent of the protective effect provided by exposure to sleep or exercise following the formation of a newly developed skill memory. For example, participation in a brief bout of cardiovascular exercise immediately after practice of a novel motor skill safeguards this new memory from a subsequent bout of training with another untrained motor skill that in the absence of the exercise will induce significant retroactive interference sufficient to eliminate memory for the initial skill (Jo et al., 2019).

Finally, it is critical to highlight that offline enhancement garnered via IP is not restricted to retention that is evaluated after $24 \mathrm{~h}$ or longer during which the learner is privy to overnight sleep as is the case for the influence of acute exercise for offline gain (Jo et al., 2019). Performance facilitation from IP has also been observed during tests administered as early as $6 \mathrm{~h}$ following the termination of practice as well as during the more common time frames used to assess memory, typically up to $72 \mathrm{~h}$, when sleeprelated consolidation might also contribute to any reported benefits (Kim and Wright, 2020). These data are important because they implicate practice organization, specifically IP, as a means to mediate postpractice consolidation independent of the powerful impact induced from exposure to sleep.

Taken as a whole, it is difficult to ignore the observation that interleaving practice of multiple novel skills as well as experiencing a period of sleep (King et al., 2017) or exercise (Robertson and Takacs, 2017) are effective means of fostering memory consolidation that can improve longterm skill retention. On the basis that functional benefits can emerge for each of these interventions, it may not be 
that surprising that evidence is emerging that suggests there may be some mechanistic overlap through which consolidation is implemented following IP, sleep, and/or exercise. These data are explored in the next sections.

\section{Consolidation of Skill Memory Involves Neural Circuitry That Includes the Primary Motor Cortex (M1)}

Consolidation responsible for the emergence of offline gain in the performance of a novel motor skill, has been argued to be dependent on, or at least involve, heightened activation of neural circuitry that includes the M1 (Robertson and Takacs, 2017; also see Mirdamadi and Block, 2020). This is certainly true in the case of early consolidation that is argued to occur in the first few hours after practice is complete. A recent proposal claims that M1 excitability shortly after the completion of procedural skill practice can be used as a physiological marker signifying that the appropriate neural machinery is in place to support offline improvement (Tunovic et al., 2014). This work demonstrated that when corticospinal excitability, typically assessed using the size of the motor-evoked potential (MEP) of the end-effector (e.g., first dorsal interosseous; FDI) induced by single-pulse transcranial magnetic stimulation (TMS) at M1, is temporarily downregulated immediately after practice, no offline gain is evident across a wake period. However, application of a theta-burst TMS protocol at this same neural site immediately after practice, designed to exogenously elevate corticospinal excitability, was sufficient to support offline improvement.

The aforementioned data have been used as the basis for a causal link between the state of $\mathrm{M} 1$ at the conclusion of training and the likelihood that offline gains will emerge from an early form of consolidation (Tunovic et al., 2014). This claim is further supported by evidence of delayed performance enhancement via the direct upregulation of M1 using non-invasive stimulation during practice of a novel skill (Reis et al., 2009). Critically, for the present discussion, the effectiveness of sleep (Xu et al., 2019) and exercise (Ostadan et al., 2016) for promoting consolidation and associated increased offline gain has also recently been associated with upregulation of cortical excitability at M1.

In keeping with the proposal that an elevation in excitability at M1 is related to the implementation of consolidation and the concomitant offline gain, there is also evidence revealing that relative greater activity of $\mathrm{M} 1$ as a significant contributor to the skill learning advantage garnered from IP over RP. Specifically, activity at M1 during response preparation is greater and occurs across a more prolonged period during a period of IP compared with RP (Lin et al., 2011). TMS-induced MEPs at the time skill memory is assessed (i.e., during delayed retention tests) are of relatively greater magnitude for learners trained with IP as opposed to RP and importantly, the magnitude of the difference in cortical excitability as a function of practice schedule correlates with extent of the offline improvement benefit observed from IP (Shea and Morgan, 1979; Lin et al., 2011 see also Tunovic et al., 2014; Fig. 1D). Moreover, applying supra-threshold single-pulse
TMS at M1 following practice trials during IP, used to disrupt processes occurring at $\mathrm{M} 1$ during training, attenuates the offline gain associated with this practice format (Lin et al., 2010). Finally, upregulating the activity at M1 during RP, via administration of anodal transcranial direct current stimulation (tDCS), can induce offline gains that are absent for participants that trained in an RP training condition but was paired with sham tDCS (Fig. 1E,F; Kim et al., 2021).

It is important to note these data do not necessarily imply that the neural substrates supporting the effects of these different interventions are (1) restricted to just M1 or (2) involve the same neural substrates. Indeed, there are recent data indicating that exogenous stimulation using tDCS of neural sites beyond M1 during RP can mediate the nature of offline changes in performance (Kim and Wright, 2020). At this juncture then, the extant data suggest that, much like the case for sleep and exercise, the implementation of consolidation following IP that moves skill memory from its initial labile to more stable or even enhanced form is linked to heightened activity at M1 or maybe more broadly in neural circuitry incorporating $M 1$. Deciphering whether this occurs from local effects at M1 or from remote influences impinging on $\mathrm{M} 1$ and whether these neural dynamics coincide across sleep, exercise, and practice organization will require additional experimental attention in the future.

\section{Final Thoughts}

Identifying training methods to foster successful longterm retention while also inducing some capability for generalization is central to research focused on the development of skill memory. Significant effort of late has been exerted in demonstrating the efficacy of "non-practice" features of the learning environment that can contribute to the continued evolution of skill memory following initial encoding. This is best reflected in the current-day interest in the role of sleep, and to a lesser extent exercise, as a means of fostering postpractice consolidation sufficient to improve offline gains in skill memory.

Addressing the potency of non-practice factors for fostering consolidation is undoubtedly important highlighting the need to expand our understanding of the entire context to which newly acquired skill memories are exposed including those that exist beyond the traditional boundaries of practice (King et al., 2017; Robertson and Takacs, 2017). This may be an especially important message on a practical level as traditionally exclusive emphasis is placed on the training or rehabilitation session itself when contemplating "best-practice." Moreover, new findings from studies addressing the contribution of sleep and exercise to offline gains in skill continue to help refine the initial mechanistic accounts of procedural skill consolidation at this time focused on the role of circuits involving M1 (King et al., 2017; Robertson and Takacs, 2017; Mirdamadi and Block, 2020). Despite these advances, the present discussion reminds us that increasing our awareness of how practice is organized, may not be such a bad thing, as it too, can foster consolidation of skill memory not afforded by other 
practice schedules (Wright et al., 2016; also see Hesseg et al., 2016).

\section{References}

Bonstrup M, Iturrate I, Thompson R, Cruciani G, Censor N, Cohen LG (2019) A rapid form of offline consolidation in skill learning. Curr Biol 29:1346-1351.

Chen J, Roig M, Wright DL (2020) Exercise reduces competition between procedural and declarative memory systems. eNeuro 7: ENEURO.0070-20.2020.

Hesseg RM, Gal C, Karni A (2016) Not quite there: skill consolidation in training by doing and observing. Learn Mem 23:189-194.

Jo JS, Chen J, Riechman S, Roig M, Wright DL (2019) The protective effects of acute cardiovascular exercise on the interference of procedural memory. Psychol Res 83:1543-1555.

Kim T, Wright DL (2020) Transcranial direct current stimulation (tDCS) of SMA complex impacts the effectiveness of interleaved and repetitive practice schedules. Neuroscience 435:58-72.

Kim T, Rhee J, Wright DL (2016) Allowing time to consolidate knowledge gained through random practice facilitates later novel motor sequence acquisition. Acta Psychol (Amst) 163:153-166.

Kim T, Chen J, Verwey WB, Wright DL (2018) Improving novel motor learning through prior high contextual interference training. Acta Psychol (Amst) 182:55-64.

Kim T, Kim H, Wright DL (2021) Improving consolidation by applying anodal transcranial direct current stimulation at primary motor cortex during repetitive practice. Neurobiol Learn Mem 178:107365.

King BR, Hoedlmoser K, Hirschauer F, Dolfen N, Albouy G (2017) Sleeping on the motor engram: the multifaceted nature of sleeprealted motor memory consolidation. Neurosci Biobehav Rev 80:1-22.

Li Y, Wright DL (2000) An assessment of the attention demands during random- and blocked-practice schedules. Q J Exp Psychol A 53:591-606.
Lin $\mathrm{CH}$, Winstein CJ, Fisher BE, Wu AD (2010) Neural correlates of the contextual interference effects in motor learning: a transcranial magnetic stimulation investigation. J Mot Behav 42:223-232.

Lin $\mathrm{CH}$, Knowlton BJ, Chiang MC, lacoboni M, Udompholkul P, Wu $A D$ (2011) Brain-behavior correlates of optimizing learning through interleaved practice. Neuroimage 56:1758-1772.

Mirdamadi JL, Block HJ (2020) Somatosensory changes associated with motor skill learning. J Neurophysiol 123:1052-1062.

Ostadan F, Centeno C, Daloze J-F, Frenn M, Lundbye-Jensen J, Roig M (2016) Changes in corticospinal excitability during consolidation predict acute exercise-induced off-line gains in procedural memory. Neurobiol Learn Mem 136:196-203.

Reis J, Schambra HM, Cohen LG, Buch ER, Fritsch B, Zarahn E, Celnik PA, Krakauer JW (2009) Noninvasive cortical stimulation enhances motor skill acquisition over multiple days through an effect on consolidation. Proc Natl Acad Sci USA 106:1590-1595.

Robertson EM, Takacs A (2017) Exercising control over memory consolidation. Trends Cogn Sci 21:310-312.

Robertson EM, Pascual-Leone A, Miall RC (2004) Current concepts in procedural consolidation. Nat Rev Neurosci 5:576-582.

Russell DM, Newell KM (2007) How persistent and general is the contextual interference effect? Res Q Exerc Sport 78:318-327.

Shea JB, Morgan RL (1979) Contextual interference effects on the acquisition, retention, and transfer of a motor skill. J Exp Psychol Hum Learn Mem 5:179-187.

Tunovic S, Press DZ, Robertson EM (2014) A physiological signal that prevents motor skill improvements during consolidation. J Neurosci 34:5302-5310.

Wright DL, Verwey W, Buchanen J, Chen J, Rhee J, Immink M (2016) Consolidating behavioral and neurophysiologic findings to explain the influence of contextual interference during motor sequence learning. Psychon Bull Rev 23:1-21.

Wulf G, Shea $\mathrm{CH}$ (2002) Principles derived from the study of simple skills do not generalize to complex skill learning. Psychon Bull Rev 9:185-211.

Xu W, De Carvalho F, Jackson A (2019) Sequential neural activity in primary motor cortex during sleep. J Neurosci 39:3698-3712. 